\title{
Improvements to aerosol attenuation measurements at the Pierre Auger Observatory
}

\author{
${\text { Max Malacari }{ }^{* a} \text { for the Pierre Auger Collaboration }}^{b}$ \\ ${ }^{a}$ Kavli Institute for Cosmological Physics, University of Chicago, Chicago, IL, USA \\ ${ }^{b}$ Observatorio Pierre Auger, Av. San Martín Norte 304, 5613 Malargüe, Argentina \\ E-mail: auger_spokespersons@fnal.gov \\ Full author list: http://www.auger.org/archive/authors_icrc_2017.html
}

\begin{abstract}
Sound knowledge of the aerosol loading in the atmosphere above the Pierre Auger Observatory is essential for the accurate reconstruction of shower energy deposit profiles using the atmospheric fluorescence technique. The vertical aerosol optical depth is inferred from hourly measurements of the vertically-fired UV laser beams of the Observatory's central laser facilities via two complementary techniques. We report on recent refinements made to these aerosol analysis techniques, and on verification of the resulting aerosol measurements through internal consistency checks using reconstructed air shower data. These analysis improvements include accounting for the shape of the aerosol scattering phase function, and the treatment of multiple scattered light from the laser beam. The cumulative effect of these improvements is a modest increase in the measured vertical aerosol optical depth above the Observatory. We will discuss the impact of this increase on the reconstruction of showers measured with the Observatory's fluorescence detector.
\end{abstract}

35th International Cosmic Ray Conference - ICRC2017

10-20 July, 2017

Bexco, Busan, Korea

\footnotetext{
*Speaker.
} 


\section{Introduction}

The Pierre Auger Observatory is a hybrid observatory for the detection of ultra-high energy cosmic rays $\left(E>10^{18} \mathrm{eV}\right)$ via the extensive air showers they produce when they interact with the earth's atmosphere [1]. It consists of a $3000 \mathrm{~km}^{2}$ surface detector (SD) array which records the lateral distribution of shower particles reaching ground level, as well as a fluorescence detector (FD) which observes the longitudinal development of showers in the atmosphere through the faint isotropic fluorescence light emitted between 300 and $420 \mathrm{~nm}$ by excited $\mathrm{N}_{2}$ molecules. The ground array is made up of 1660 water-Cherenkov detectors arranged on a triangular grid with a spacing of $1.5 \mathrm{~km}$. It is overlooked from its periphery by 27 fluorescence telescopes located at 4 sites.

While the SD records the footprints of extensive air showers with a duty cycle of almost $100 \%$, the energy scale of the Observatory is determined using coincident observations of a subset of these showers with the fluorescence detector (having a duty cycle of $\sim 15 \%$ [1]), which provides a near-calorimetric measurement of the shower energy. As this fluorescence light can travel tens of kilometres between its point of emission and a fluorescence detector, it is essential that the transmission properties of the atmosphere are well understood [2]. In the lowest $15 \mathrm{~km}$ of the atmosphere where air shower measurements occur, aerosols play an important role in modifying the light transmission. While the UV extinction due to aerosols is several times less than the extinction due to molecules, the aerosol atmosphere is highly variable over short time-scales and, on hazy nights, the light flux from distant showers can be reduced by a factor of 3 or more due to aerosol attenuation.

\section{Measurement of the vertical aerosol optical depth}

Measurements of vertical laser tracks from the Observatory's Central and eXtreme Laser Facilities (CLF and XLF) are used to infer the vertical distribution of aerosols above the array with a time resolution of one hour via two independent and complementary techniques [3, 4]. Sets of 50 vertical depolarized $355 \mathrm{~nm}$ laser pulses are measured every 15 minutes at each of the 4 FD sites during each night of observation. To minimize the statistical uncertainty in the measured laser signal, each set of measurements within an hour block is averaged before being used to calculate the Vertical Aerosol Optical Depth (VAOD), the integral of the aerosol attenuation coefficient $\alpha_{\mathrm{A}}$ from the ground to height $h$, within the field of view of each of the FD sites. In the Data Normalized (DN) method, the hourly averaged laser traces are compared to averages collected under nominally aerosol-free conditions (called reference nights ${ }^{1}$ ), and in the Laser Simulation (LS) method, the average traces are compared to simulations generated under different aerosol attenuation conditions. Hourly VAOD profiles for each FD site are written to an aerosol database which is queried during air shower reconstruction to determine the aerosol attenuation between any two points in the atmosphere above the array.

The DN method is the primary technique used to retrieve the VAOD, and aerosol profiles calculated in this way comprise $\sim 90 \%$ of the database. The fundamental equation for the calculation of the VAOD using this method, which can be analytically derived under the assumptions that the

\footnotetext{
${ }^{1}$ Typically a new reference night is chosen each year.
} 


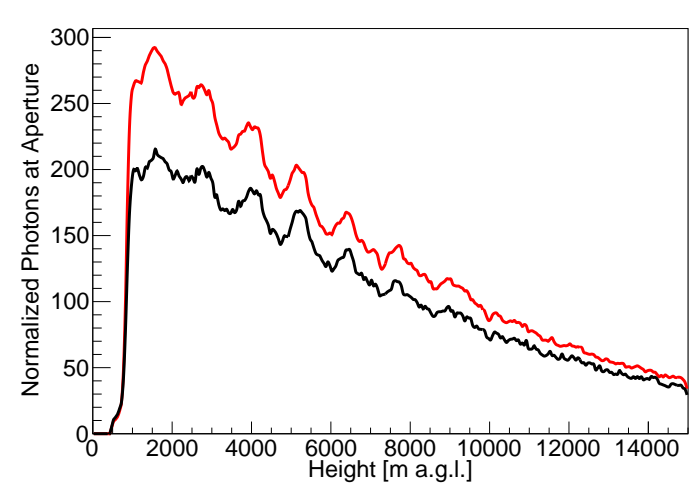

(a)

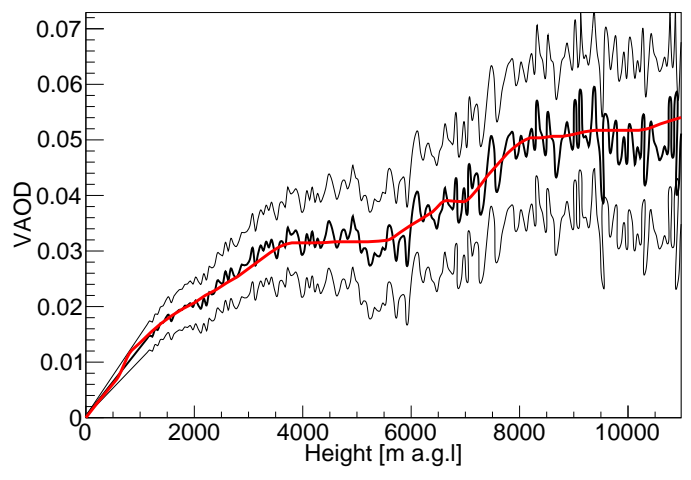

(b)

Figure 1: (a) Example of a measured laser trace (black). In red is the associated nominally clear reference trace. (b) The VAOD as derived from this measurement. The central black profile is the raw VAOD, and in red is a smoothed version. The upper and lower black profiles denote the raw upper and lower uncertainty bounds on the VAOD.

aerosol atmosphere is horizontally uniform, and that multiple scattered light from the laser does not contribute to the light flux measured at the FD, is

$$
\operatorname{VAOD}(h)=\underbrace{\frac{-1}{1+1 / \sin \phi_{2}} \ln \left(\frac{N_{\mathrm{aer}}}{N_{\mathrm{ref}}}\right)}_{(1)}+\underbrace{\frac{1}{1+1 / \sin \phi_{2}} \ln \left(1+S_{\mathrm{A}} / S_{\mathrm{M}}\right)}_{(2)} .
$$

Here $N_{\text {aer }}$ and $N_{\text {ref }}$ are the photon fluxes originating from height $h$ (on the night of measurement and the reference night respectively), $\phi_{2}$ is the elevation of the laser track segment with respect to the detector, and $S_{\mathrm{A}}$ and $S_{\mathrm{M}}$ describe the fraction of the laser beam scattered towards the detector from that height by aerosols and molecules respectively. Terms (1) and (2) of Eq. 2.1 both encode separate pieces of information about the aerosol attenuation properties of the atmosphere, with the first part representing the transmission of laser light along the laser beam to height $h$, and from that point to the aperture of a fluorescence detector, and the second representing the scattering of laser light out of the beam and towards the detector. Traditionally the analysis operates under an additional assumption; that the density of molecular scattering centres in the atmosphere is much greater than that of aerosols. This, coupled with the fact that the aerosol scattering phase function is strongly forward peaked and the vertical laser beam is always viewed nearly side on, means that the $S_{\mathrm{A}}$ term is much smaller than the $S_{\mathrm{M}}$ term. In this case, term (2) $\rightarrow 0$ and the VAOD at a given height depends only on the ratio of the measured light flux originating from that height relative to that measured on the reference night. An example of a measured averaged laser trace, along with a reference trace, is shown in Fig. 1a. In Fig. $1 \mathrm{~b}$ is the corresponding reconstructed VAOD determined using the DN technique. The average VAOD at $3 \mathrm{~km}$ above ground level (above the planetary boundary layer) measured at the Observatory site is approximately 0.04 [2].

\section{Improvements to the analyses}

The two aerosol analyses have been updated to remove their reliance on a number of simpli- 


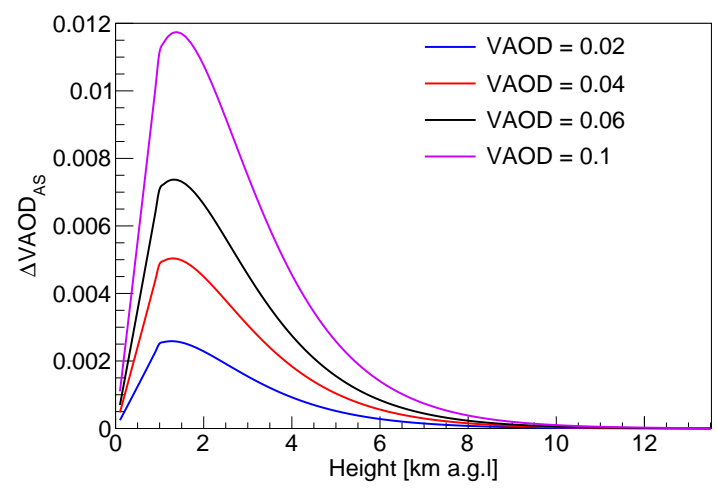

(a)

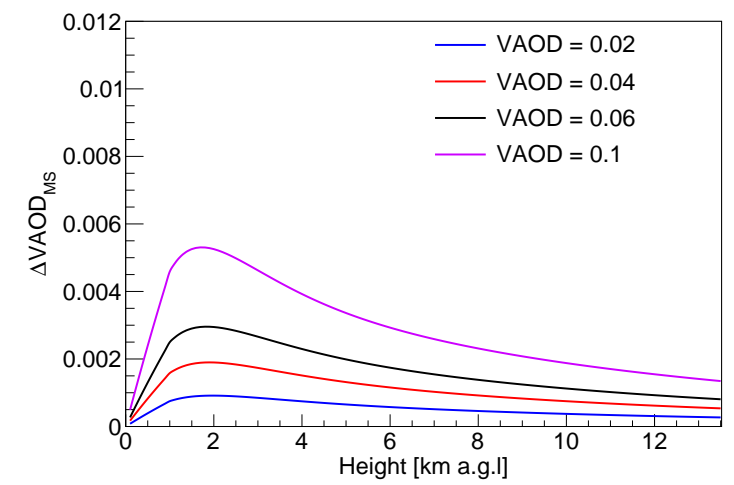

(b)

Figure 2: (a) Correction for aerosol scattering out of the laser beam in the DN analysis. (b) Correction for the small multiple scattered component of the received laser light flux at the detector.

fying assumptions which are described here.

\subsection{Correction for aerosol scattering}

The DN analysis previously operated under the assumption that scattering out of the laser beam towards the fluorescence detector is due to the molecular component of the atmosphere only.

Term (2) in Eq. 2.1 can be considered as the correction term taking into account aerosol scattering of photons out of the laser beam. Since $S_{\mathrm{A}}$ and $S_{\mathrm{M}}$ are always $\geq 0$, this term is always positive and hence serves to increase the VAOD. Physically speaking, increasing $S_{\mathrm{A}}$ is akin to increasing the expected photon flux at the detector (as we now expect to measure both molecular and aerosol scattered light), and as the measured light flux is fixed, this will have the effect of decreasing the apparent aerosol transmission.

An analytical approximation to the magnitude of this correction as a function of height is shown in Fig. 2a. In this model the aerosol atmosphere is approximated using a simple 3-parameter exponential with a planetary boundary layer of uniform aerosol density. The thickness of the mixing layer and the scale height are fixed, while the aerosol horizontal attenuation length at ground level is varied to emulate a range of typical VAODs at the top of the atmosphere. The correction has the largest effect low in the atmosphere where the aerosol concentration is highest. The scale height of the molecular atmosphere is typically several times larger than the scale height of the aerosol atmosphere, so the ratio of the number of aerosol to molecular scattering centres decreases rapidly with height. This, coupled with the very small aerosol phase function component at scattering angles $>90^{\circ}$ means that the correction drops off quickly above its height of maximum amplitude.

In practice the quantity $S_{\mathrm{A}}$ is not known a priori as it relies on knowledge of the aerosol scattering coefficient at each height. The correction is therefore applied iteratively by assuming that $S_{\mathrm{A}} \ll S_{\mathrm{M}}$, calculating the aerosol scattering coefficient from the resultant VAOD profile, and then recalculating the VAOD profile using Eq. 2.1.

\subsection{Correction for multiple scattering}

In both VAOD reconstruction techniques it was previously assumed that any light that is not 
initially scattered out of the laser beam and towards the detector is lost. This assumption ignored the possibility that some photons will reach the detector after one or more additional scatterings. As multiple scattering serves to make the laser appear slightly brighter than it would under the assumption of single scattering only, both of these VAOD reconstruction methods compensated for this effect by reconstructing an aerosol atmosphere that was marginally cleaner than reality.

The DN and LS aerosol analyses can be corrected for multiple scattering by effectively subtracting the estimated multiple scattered contribution from the total light flux in each height bin. For the DN analysis this has the form

$$
\frac{N_{\text {aer }}}{N_{\text {ref }}} \rightarrow \frac{N_{\text {aer }}}{N_{\text {ref }}} \frac{1-f_{\text {aer }}}{1-f_{\text {ref }}},
$$

where $f_{\text {aer }}$ and $f_{\text {ref }}$ denote the fraction of multiple scattered light received in a given signal time bin relative to the total (on the night of measurement and the reference night respectively). By substituting this expression into term (1) of Eq. 2.1 the VAOD can be corrected for multiple scattering of the laser beam.

The quantity $f$ is parametrized using Monte Carlo raytracing simulations of a vertical $355 \mathrm{~nm}$ laser beam and has the form

$$
f_{\zeta}(\alpha, \tau)=k \alpha^{A} \tau
$$

where $\alpha$ denotes the total volume scattering coefficient at the laser track (aerosol and molecular, in $\mathrm{m}^{-1}$ ) in a given time bin, and $\tau$ is the total optical depth between the fluorescence detector and the laser track segment [5]. The parameter $\zeta$ denotes the angular size (in degrees) of the region on the focal surface of the detector (centred on the laser's track across the camera) over which light is integrated in each signal time bin. For analyses of vertical laser shots $\zeta$ is fixed to $1.5^{\circ}$, and the parameters $k$ and $A$ are 12.98 and 0.57 respectively. Fig. 3a shows the flux of photons at the detector's focal surface as a function of direction taken from the raytracing simulation, and Fig. $3 \mathrm{~b}$ shows the fitted parametrization for an integration angle of $1.5^{\circ}$.

The expected magnitude of the VAOD increase as a function of height above ground level based on simulations is shown in Fig. 2b. In practice the correction must be applied iteratively to the DN analysis, as the aerosol scattering coefficient at the laser track and the aerosol optical depth between the laser track and the detector, necessary for the calculation of $f_{\zeta}$, are unknown a priori. In the case of the LS analysis, the multiple scattered fraction can simply be added to the simulated light flux at the detector before comparison with a measured laser trace.

\subsection{Measurement of the aerosol scattering phase function}

Both of the improvements made to the aerosol analysis which are described here have a dependence on the shape of both the molecular and aerosol scattering phase functions. The shape of the aerosol scattering phase function can not be calculated analytically, and depends on the size and shape distributions of the aerosols present in the atmosphere, which can vary over short timescales. The shape of the aerosol phase function at a wavelength of $350 \mathrm{~nm}$ (close to the CLF and XLF wavelength) is measured hourly at the Observatory during FD data taking, using observations of a xenon flasher fired horizontally across the field of view of two of the fluorescence detectors [6]. 


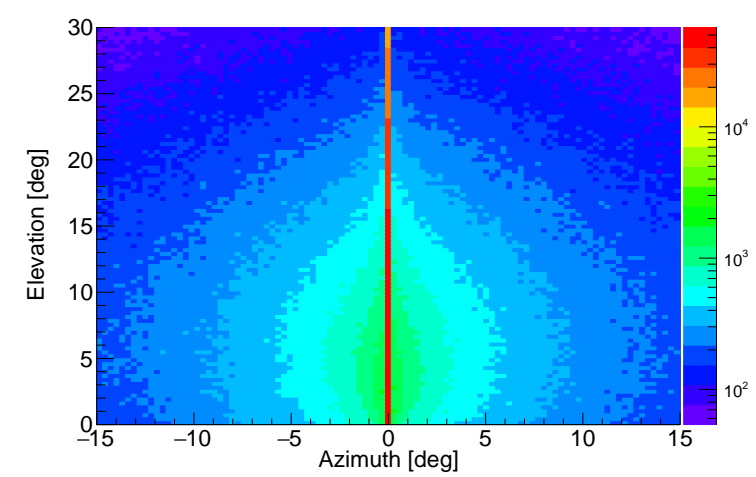

(a)

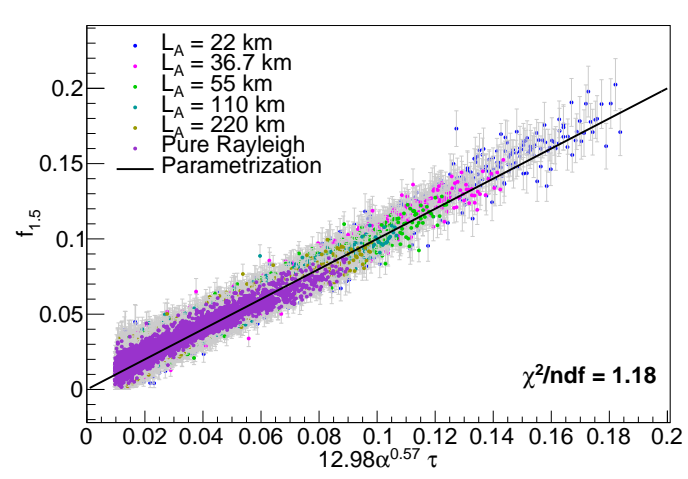

(b)

Figure 3: (a) Simulated distribution of light at the focal surface of the FD (photons at the aperture) from a vertical laser beam (integrated over time). The intense red stripe at an azimuth of $0^{\circ}$ corresponds to single scattered photons from the laser beam. All other photons are multiple scattered. (b) Simulated multiple scattered fraction within $\zeta=1.5^{\circ}$ for a range of aerosol attenuation conditions overlaid with the parametrization $f_{\zeta}(\alpha, \tau)$.

The form of the aerosol scattering phase function used for calculations at the Pierre Auger Observatory is the modified Henyey-Greenstein phase function

$$
\left(\frac{1}{\sigma} \frac{\mathrm{d} \sigma}{\mathrm{d} \Omega}\right)_{\mathrm{A}}=\frac{1-g^{2}}{4 \pi}\left(\frac{1}{\left(1+g^{2}-2 g \cos \theta\right)^{3 / 2}}+f \frac{3 \cos ^{2} \theta-1}{2\left(1+g^{2}\right)^{3 / 2}}\right),
$$

which describes the fraction of light per unit solid angle that is scattered in a particular direction by aerosols. The Henyey-Greenstein phase function includes two parameters, $f$ and $g$, known as the backscattering and asymmetry parameters, which describe its shape. For a backscattering parameter of $f=0$ the asymmetry parameter is simply equal to the mean cosine of the scattering angle. The nominal values for these parameters at the Auger site are $g=0.6 \pm 0.1$ and $f=0.4$ [2].

The improvements to the aerosol analysis described in this contribution both have a dependence on the aerosol phase function shape, in particular the asymmetry parameter $g$. The shape of the aerosol scattering phase function is expected to have some seasonal variation, as the size and shape distributions of aerosol particles above the array change throughout the year [7]. Approximately 2100 hours of xenon flasher measurements taken at the Coihueco FD site between 2011 and 2015 were analyzed to search for significant departures of the asymmetry parameter from its average value throughout the year. Fig. 4 a shows the mean value of $g$ measured over this 5-year dataset, which is in agreement with the previously published value of this parameter [2]. Fig. 4b shows the average asymmetry parameter as a function of the time of year. A small seasonal dependence is evident, however the drift in the parameter throughout the year is less than the root-mean-square spread within any single month. Therefore $g$ is set to its nominal value of 0.6 for both of the aerosol analysis improvements described here.

\section{Effect on reconstructed air showers}

The height-dependence of these aerosol analysis improvements results in the alteration of re- 


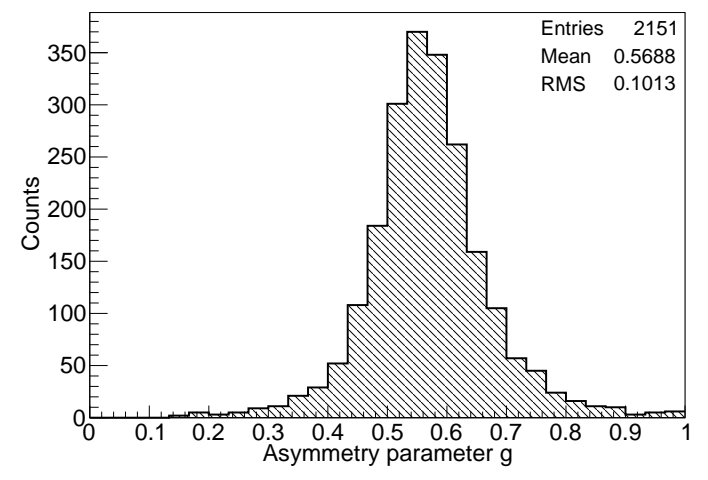

(a)

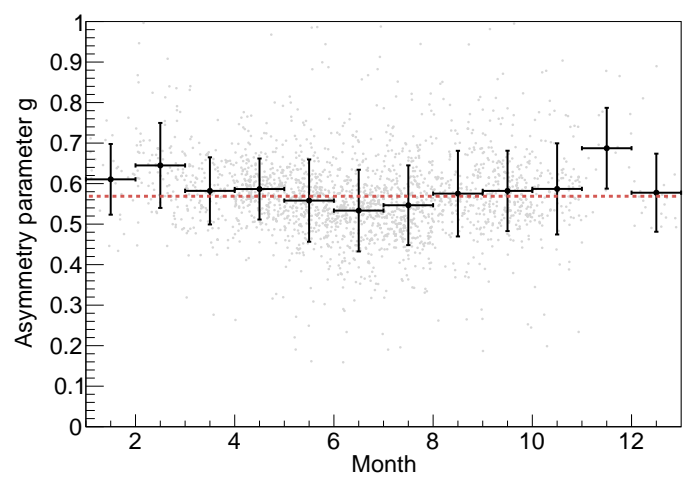

(b)

Figure 4: (a) Distribution of the fitted asymmetry parameter over the 5-year dataset. (b) Drift in the asymmetry parameter throughout the year. Vertical error bars indicate the root-mean-square of the fitted asymmetry parameter distribution in each month of data. The horizontal line indicates the average value over the whole dataset.

constructed shower profiles in both shape and normalization. Reconstructed shower energies are increased on average by $1.5 \%$ at $10^{17.5} \mathrm{eV}$, up to $3 \%$ at an energy of $10^{19.5} \mathrm{eV}$. Showers of higher energy tend to be detected at larger distances, meaning the aerosol transmission is lower, and hence the relative decrease in the aerosol transmission under these aerosol analysis improvements is larger. Changes in the average depth of shower maximum are driven by the elongation of the decaying tail of shower profiles and range from $2 \mathrm{~g} / \mathrm{cm}^{2}$ at $10^{17.5} \mathrm{eV}$, up to $5 \mathrm{~g} / \mathrm{cm}^{2}$ at an energy of $10^{19.5} \mathrm{eV}$. The larger VAOD increases close to the ground (shown in Fig. 2) lead to a modest elongation of the trailing edge of reconstructed energy deposit profiles, shifting their depth of maximum development slightly deeper in the atmosphere. Both the energy and $X_{\max }$ increases are small on average, and are within current aerosol transmission related systematic uncertainties in the energy and $X_{\max }$ scales published in [8,9].

A useful metric for verifying the validity of the aerosol attenuation measurements used in shower reconstruction is the ratio $E_{\mathrm{SD}} / E_{\mathrm{FD}}$, the ratio of reconstructed $\mathrm{SD}$ to $\mathrm{FD}$ energy. If the aerosol loading above the array is characterized correctly we should see internal consistency within our reconstructed air shower data, and there should be no dependence of this ratio on the aerosol transmission to the depth of maximum shower development, which functions as a proxy for the distance of the shower from the FD (the reconstructed SD energy is independent of the distance of the shower from the fluorescence detector). If the relationship exhibits a negative slope it indicates that showers observed at larger distances to the FD are being reconstructed with lower energies on average, suggesting that the aerosol content in the atmosphere has been underestimated (Fig. 5a). Conversely, a positive slope to the relationship would indicate an overcorrection for aerosol attenuation. Following the improvements to our aerosol analysis techniques, we see a slope in this ratio of $-0.006 \pm 0.036$ (Fig. 5b), fully consistent with zero, and a strong indication that our VAOD measurements accurately describe the status of the aerosol atmosphere above the array. The sensitivity of the slope to uncertainties in the aerosol concentration is currently being assessed. 


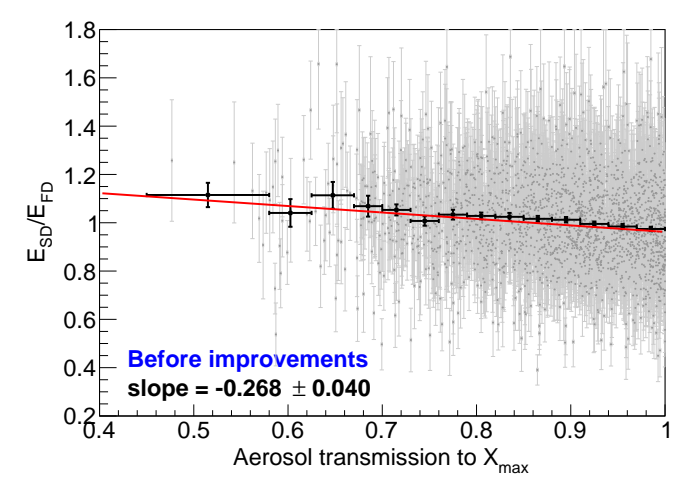

(a)

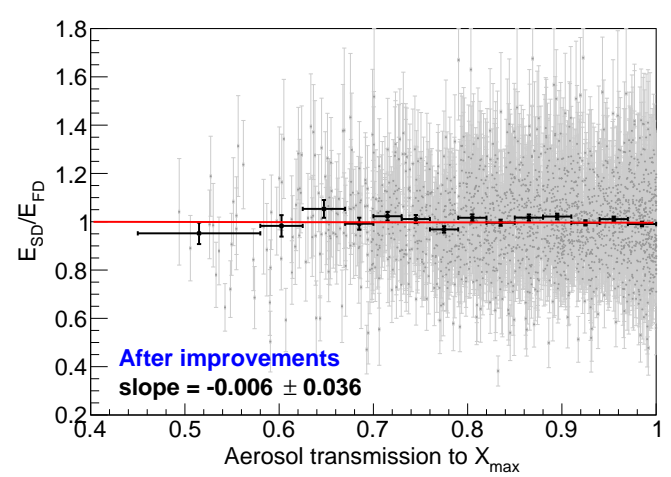

(b)

Figure 5: Ratio of the reconstructed SD to FD energy as a function of the aerosol transmission to the depth of shower maximum. a) Before the improvements made to aerosol extinction measurements. The negative slope indicates that the aerosol content of the atmosphere has been underestimated. b) Following the improvements made to aerosol extinction measurements. The slope is fully compatible with zero, demonstrating internal consistency within the data.

\section{Conclusion}

Two simplifying assumptions used in the calculation of the aerosol transmission properties of the atmosphere above the Pierre Auger Observatory have been removed. These refinements include properly accounting for the aerosol side-scattering and multiple scattering of light from the Observatory's two laser facilities, which are used to make hourly measurements of the vertical aerosol loading above the array.

These analysis improvements increase the reconstructed aerosol concentration close to the ground on average, resulting in modest energy-dependent increases in the reconstructed energy and depth of maximum of air showers measured with the Observatory's fluorescence detector, which are within our current aerosol related systematic uncertainties. In addition, we see internal consistency between shower energies reconstructed with both the surface and fluorescence detector, indicating a sound knowledge of the aerosol loading in the atmosphere above the Observatory.

\section{References}

[1] The Pierre Auger Collaboration, Nucl. Instr. Meth. A 798 (2015) 172-213.

[2] The Pierre Auger Collaboration, Astropart. Phys. 33 (2010) 108-129.

[3] B. Fick et al., JINST 1 (2006) P11003.

[4] The Pierre Auger Collaboration, JINST 8 (2013) P04009.

[5] M. Malacari and B.R. Dawson, Astropart. Phys. 93 (2017) 38-45.

[6] S.Y. BenZvi et al., Astropart. Phys. 28 (2007) 312-320.

[7] The Pierre Auger Collaboration, Atmos. Res. 149 (2014) 120-135.

[8] V. Verzi for The Pierre Auger Collaboration, Proc. 33rd Int. Cosmic Ray Conf. (2013).

[9] The Pierre Auger Collaboration, Phys. Rev. D 90 (2014) 122005. 